\title{
Impurity concentration dependent electrical conduction in germanium crystals at low temperatures
}

\author{
MANORANJAN GHOSH*, SHREYAS PITALE, S G SINGH, SHASHWATI SEN and S C GADKARI \\ Crystal Technology Section, Technical Physics Division, Bhabha Atomic Research Centre, Trombay, Mumbai 400088, \\ India \\ *Author for correspondence (mghosh@barc.gov.in)
}

MS received 18 December 2018; accepted 21 February 2019

\begin{abstract}
A germanium single crystal of $7 \mathrm{~N}+$ purity with a diameter of $45 \mathrm{~mm}$ and a length of $100 \mathrm{~mm}$ has been grown by the Czochralski method. The structural quality of the crystal has been characterized by Laue diffraction. Electrical conduction and Hall measurements are carried out on samples retrieved from different parts of the crystal along the growth axis. The top part of the crystal exhibits the lowest impurity concentration $\left(\sim 10^{12} \mathrm{~cm}^{-3}\right)$ that gradually increases towards the bottom $\left(10^{13} \mathrm{~cm}^{-3}\right)$. The crystal is n-type at room temperature and the resistivity shows a non-monotonic temperature dependence. There is a transition from n-type to p-type conductivity below room temperature at which bulk resistivity shows a maximum and dip in carrier mobility. The intrinsic to extrinsic transition region shifts towards room temperature as the impurity concentration increases and it reflects the purity level of the crystal. A similar trend is observed in the boron-implanted high purity germanium (HPGe) crystal at different doping levels. The phenomena can be understood as a result of interplay between a temperature dependent conduction mechanism driven by an impurity band and an intrinsic carrier in Ge crystals having fairly low acceptor concentrations $\left(<10^{12} \mathrm{~cm}^{-3}\right)$.
\end{abstract}

Keywords. Ge crystal growth; minority carrier lifetime; resistivity; carrier concentration; hall mobility; boron implantation.

\section{Introduction}

Germanium is a group VI indirect semiconductor like silicon but with a smaller band gap and higher carrier mobility [1,2]. The purest form of germanium shows high resistivity and high electron and hole mobility at low temperature. These properties make $\mathrm{Ge}$ more attractive than $\mathrm{Si}$ for optoelectronic applications. However, the presence of an unstable oxide layer at the surface restricts its application in electronic devices. Relatively low purity crystals find applications in waveguides, infrared (IR) window fibre optics, IR night vision devices, space solar cells and as polymerization catalysts [3]. High purity $\mathrm{Ge}$ (HPGe) crystals are used for the fabrication of a thick gamma ray detector [4,5]. Therefore, the growth of the pure germanium crystal is highly desirable, especially for nuclear detector application [6-8]. Single crystals of germanium are grown at various purity levels. In particular, the purification of the raw material and growth of HPGe remain a challenging task for many laboratories [9-12]. The impurities present herein (concentration of $\sim 10^{10} \mathrm{~cm}^{-3}$ ) are primarily unintentional. Doping of selective dopants is also performed for the fabrication of the detector and other electronic devices $[13,14]$. Therefore, it is important to understand the electrical conduction properties of $\mathrm{Ge}$ at various purity levels.
The temperature dependent transport properties of germanium are highly sensitive to its purity level [15]. Germanium, having an impurity concentration above $10^{15} \mathrm{~cm}^{-3}$, shows anomalies in resistivity, hall coefficient and mobility at low temperature [16]. It is proposed that impurity band conduction is primarily responsible for such behaviour [17]. There are limited studies on the conduction properties of Ge with impurity concentration ranging from $10^{10}$ to $10^{15} \mathrm{~cm}^{-3}$. Also, the type of conductivity (impurity) has a visible impact on the conduction properties. In a few studies with a higher level of impurity concentration, Ge crystals do not exhibit a change in type of conductivity throughout the temperature range [16]. In some studies, crystals with lower impurity concentration show n-type at higher temperature and p-type at low temperature [18]. Resistivity and mobility curves also show different behaviours when such transition occurs. Therefore, exhaustive studies on the transport properties of Ge with a lower level of impurity concentration are relevant.

In this study, the $7 \mathrm{~N}$ pure Ge crystal is grown and characterized. The transport properties of Ge crystals with impurity concentration in the range of $10^{10}-10^{14} \mathrm{~cm}^{-3}$ have been investigated. p-type Ge crystals of such purity change their type of conductivity with associated changes in resistivity 
and mobility at certain temperature. It is observed that the transition in type of conductivity occurs at higher temperature when impurity concentration increases.
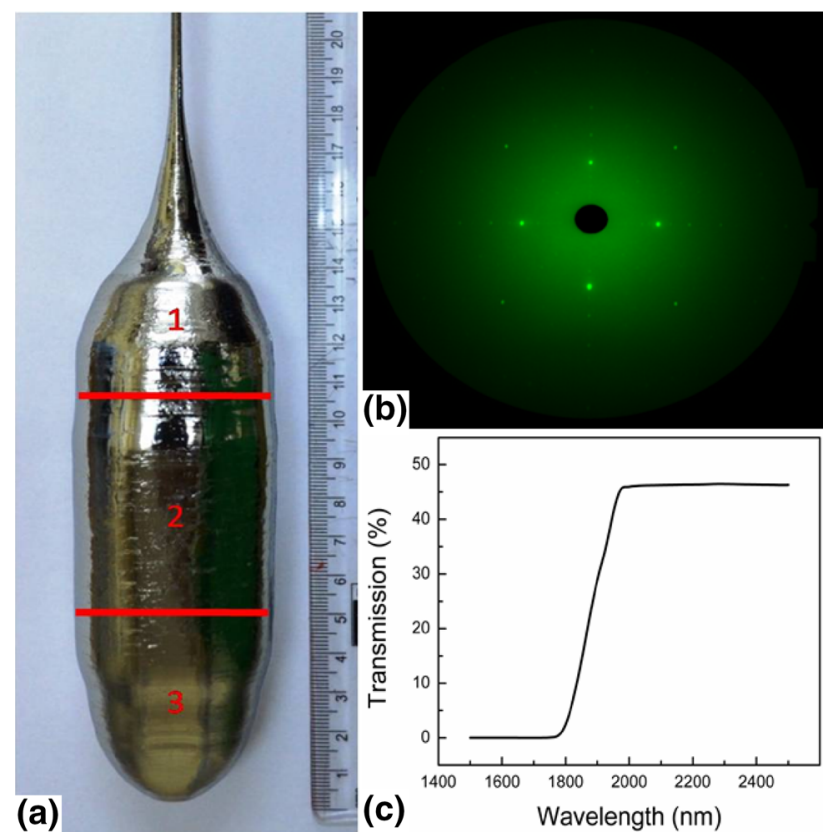

Figure 1. (a) $7 \mathrm{~N}$ pure as a grown germanium single crystal, (b) Laue diffraction pattern of a grown crystal and (c) transmission spectra of a $5 \mathrm{~mm}$ thick germanium cut from the grown crystal.

\section{Growth and processing of Ge crystals}

A germanium-single crystal with a diameter of $45 \mathrm{~mm}$ and a length of $100 \mathrm{~mm}$ of $7 \mathrm{~N}+$ purity has been grown by the Czochralski method (figure 1a) from a zone-refined polycrystalline ingot (weight $850 \mathrm{~g}$ ) supplied by the Chemistry Division, BARC. The charge consisting of zone-refined ingot is etched in concentrated $\mathrm{HNO}_{3}(65 \%): \mathrm{HF}(48 \%):: 3: 1$ solution for $10 \mathrm{~min}$, rinsed under running deionized (DI) water $\left(>18 \mathrm{M} \Omega\right.$ ) and dried under high pure $\mathrm{N}_{2}$. Then the material is placed in a quartz crucible (cleaned by dipping in $10 \%$ $\mathrm{HF}$ and rinsing in $\mathrm{DI} \mathrm{H}_{2} \mathrm{O}$ ) with a diameter of $100 \mathrm{~mm}$ and a length of $150 \mathrm{~mm}$. The seed crystal (oriented towards the $\langle 100\rangle$ direction) was cleaned and etched by following similar steps as mentioned above and used for the crystal growth. A graphite susceptor is coupled with a $50 \mathrm{~kW}, 10-13 \mathrm{~Hz}$ radio frequency (RF) generator to melt the material. However, it is found that at high temperature germanium gets coupled with the RF, thus making the seeding procedure difficult. This problem was avoided by adjusting the melt height. A typical rotation rate of $15-20 \mathrm{rpm}$ and a pull rate of $20-25 \mathrm{~mm} \mathrm{~h}^{-1}$ are used during the growth of the Ge-single crystal. The growth experiments are carried out in a class- 10000 clean room while material preparation is performed in a class- 1000 clean environment. The purity of the grown crystal is comparable to that of the initial charge (polycrystalline zone refined ingot) as verified by analytical techniques (data not shown). Thus, no extra impurity was added during the crystal growth process. (a)

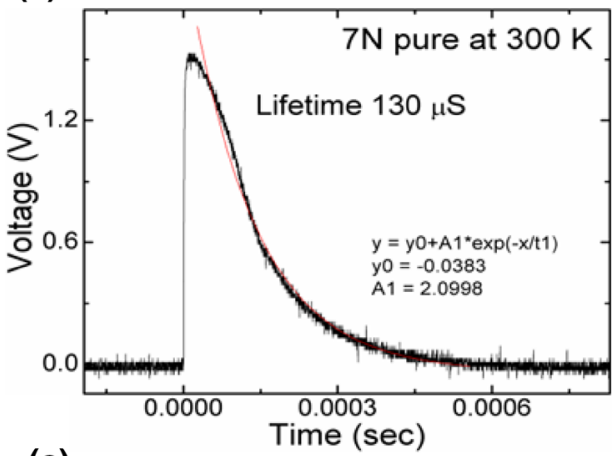

(c)

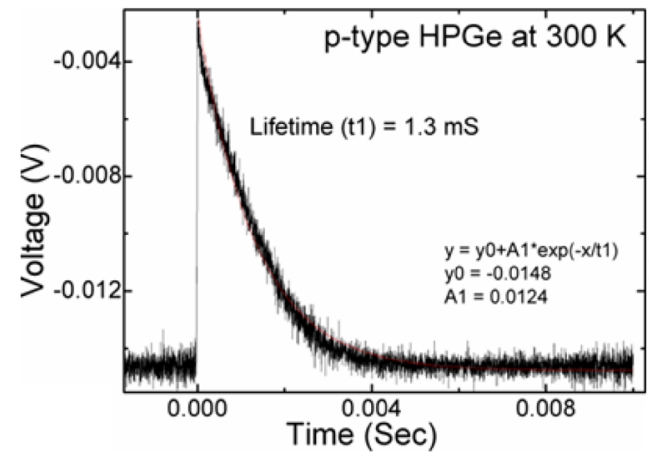

(b)

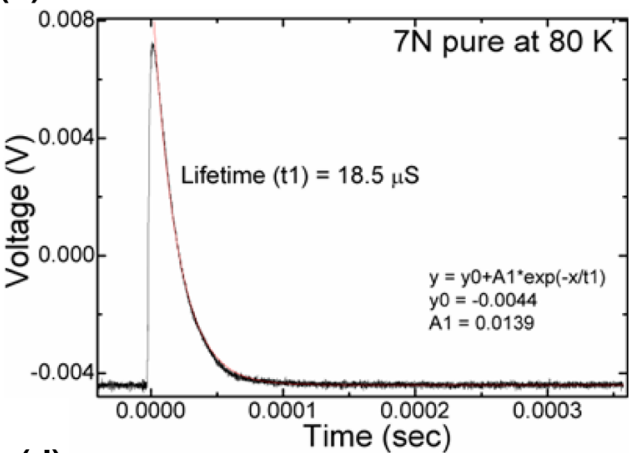

(d)

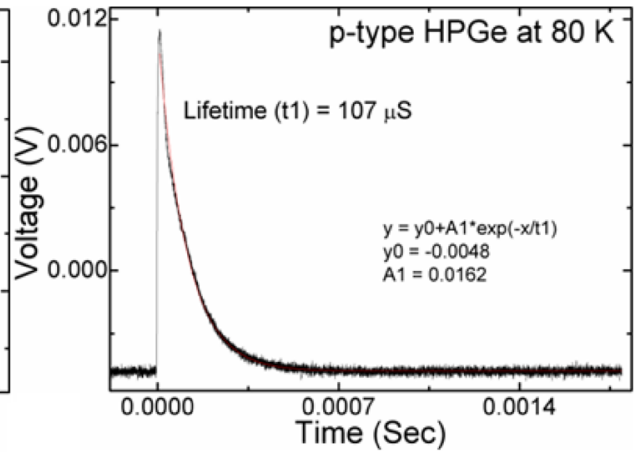

Figure 2. MCLTs of a grown germanium $(\mathbf{a}, \mathbf{b})$ and p-type HPGe $(\mathbf{c}, \mathbf{d})$ crystal as indicated in the view graph at 300 and $80 \mathrm{~K}$. Red lines indicate a single exponential fitting of experimental data. 
The grown crystal was cut into slices of a desirable size by a diamond wire saw cutting machine. Then the slices are further cut into pieces of intended size and shape. All the pieces were subsequently ground and lapped. Lapping is performed in steps by silicon carbide abrasive paper with different grit sizes (600-1500). All pieces are polished by diamond paste of $10,5,3$ and $0.3 \mu \mathrm{m}$ particle sizes to remove lapping imperfections and to achieve a smooth mirror finish surface. Crystals are further cleaned using ultrasonication in suitable solvents to remove organic and inorganic impurities from the surface. Finally, the crystal slices are etched before the formation of electrical contacts. The crystals are stored in an inert atmosphere glove box for further use.

Structural characterization of the crystal is carried out by Laue back-reflection and transmission measurements (1500$2500 \mathrm{~nm}$ ). For this purpose, the grown crystal is cut into $5 \mathrm{~mm}$ thickness and with varying cross sections $\left(5 \times 5 \mathrm{~mm}^{2}\right.$ for structural measurement and $35 \times 35 \mathrm{~mm}^{2}$ for optical measurement). The Laue back-reflection pattern (recorded using a Bruker make Laue camera) of the grown crystal is shown in figure $1 \mathrm{~b}$. The $\langle 100\rangle$ orientation of the grown crystal was confirmed by fitting the back-reflection pattern using the Orient-express

(a)
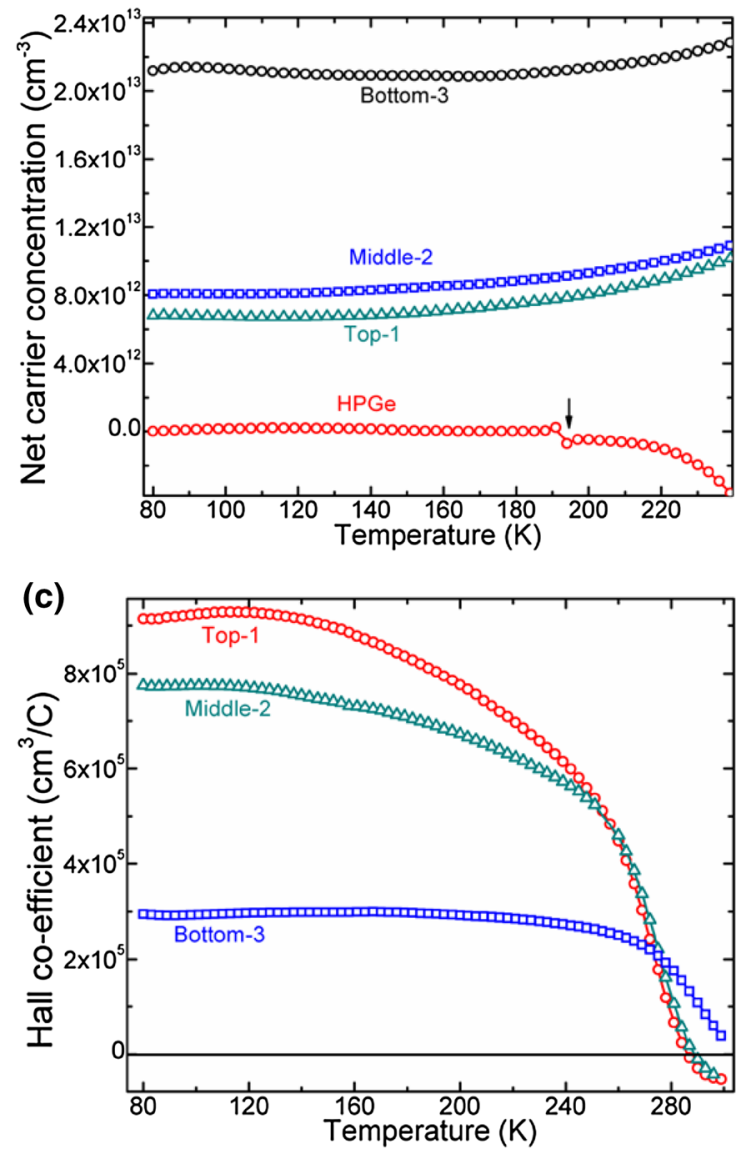

code. The transmission spectra recorded (by the Shimadzu 3600 spectrophotometer) for the Ge sample $\left(35 \times 35 \times 5 \mathrm{~mm}^{3}\right)$ is shown in figure 1c. The optical band gap calculated from the transmission data is found to be $\approx 0.70 \mathrm{eV}$.

\section{MCLT measurement}

The residual impurity concentration in the HPGe crystal is beyond the detection limit of standard analytical techniques. Physical characterization techniques like Hall measurement and minority carrier lifetime (MCLT) are carried out to understand the transport and recombination of carriers in $\mathrm{Ge}$ crystals.

Lifetime measurements are found useful for determining the presence of special types of crystal imperfections, which are usually present in minuscule amounts that cannot be detected by Hall or resistivity measurements [19]. The degree of crystal perfection is determined in terms of the maximum value of the carrier lifetime [20]. Knowledge of the average carrier lifetime gives an estimation of the purity level of crystals grown in this laboratory. The laboratory grown

(b)
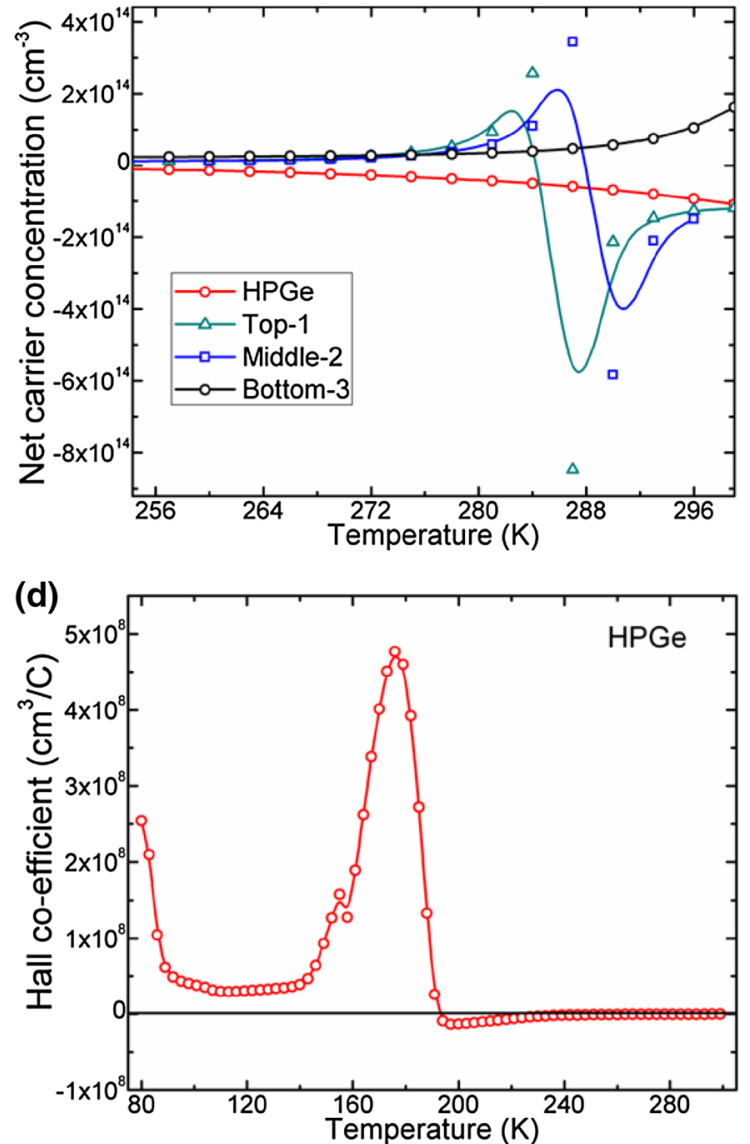

Figure 3. (a) Temperature dependent net carrier concentration of HPGe and different parts of the $7 \mathrm{~N}$ pure germanium crystal, (b) expanded view of the same near room temperature. Hall coefficients of: (c) $7 \mathrm{~N}$ pure germanium and (d) HPGe crystals. 
germanium crystal and Umicore HPGe (both p and n-type) crystals are characterized by MCLT measurement using the Edinburgh FLP 920 instrument and a Keithley current source (Model: 6221). The samples were mounted in an Oxford make optical cryostat and excited using a pulsed xenon source at a frequency of $50 \mathrm{~Hz}$.

Signals were recorded on a Tektronix digital oscilloscope. Measurements were performed both at room temperature and $80 \mathrm{~K}$.

The steady-state equilibrium of charge carriers is disturbed and excess minority charge carriers are generated by optical excitation using a pulsed xenon lamp. The majority charge carriers are then drawn into the sample from an external current source to preserve space charge neutrality. The resultant effect is the excess density of both charge carriers. After the removal of disturbance, the excess charge carriers establish equilibrium through various processes. The decay curves recorded for $7 \mathrm{~N}$ pure germanium and HPGe crystals are shown in figure $2 \mathrm{a}-\mathrm{d}$, respectively. Carrier lifetimes are extracted by single exponential fitting of the spectra. The lifetime thus measured for the Umicore HPGe crystal (figure 2c and d) corroborates well with the supplier's data sheet. Usually, the crystal with a longer carrier lifetime is considered a better candidate for the fabrication of the $\mathrm{p}-\mathrm{i}-\mathrm{n}$ diode. A lifetime recorded at room temperature is nearly 10 times higher than that measured at $77 \mathrm{~K}$. The room temperature lifetime of $7 \mathrm{~N}$ pure Ge is found to be $130 \mu \mathrm{s}$. On the other hand, an $\mathrm{HPGe}$ crystal shows a lifetime $>1 \mathrm{~ms}$.

\section{Temperature-dependent Hall measurement by the van der Pauw method}

Basic semiconductor properties of Ge are studied by fourprobe resistivity measurement using the van der Pauw method [21,22]. Parameters such as resistivity, impurity concentration and carrier mobility are determined in this study by temperature dependent Hall measurements conducted using an Ecopia make HMS5000 Hall measurement system. Ge slices were cut from three sections of the grown crystal viz. top, middle and bottom parts as indicated in figure 1a. Hall measurements were performed according to the procedure described in the IEEE standard [23] on samples from each region appearing lamellar and square in shape with the shortest linear dimension along a face being at least three times greater than the thickness. Surface roughness and strains (such as those caused by lapping) were removed by polishing and etching. Four contacts were attached on the corners of polishetched square samples at $90^{\circ}$ spacing using an In-Ga eutectic.

The net carrier concentration and Hall coefficient are plotted as a function of temperature in figure 3a-d. An HPGe crystal at $77 \mathrm{~K}$ exhibits p-type conductivity with a measured carrier concentration of $8.4 \times 10^{9} \mathrm{~cm}^{-3}$. The top part of the $7 \mathrm{~N}$ pure $\mathrm{Ge}$ crystal also shows p-type conductivity with a higher carrier concentration of $7.1713 \times 10^{12} \mathrm{~cm}^{-3}$

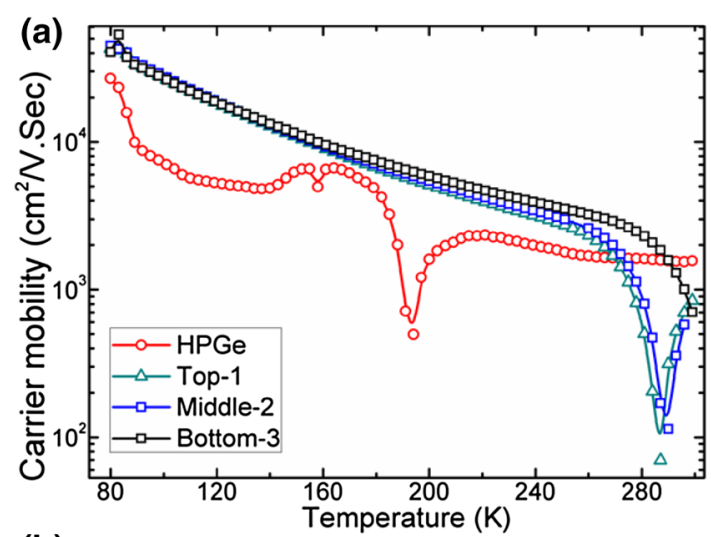

(b)

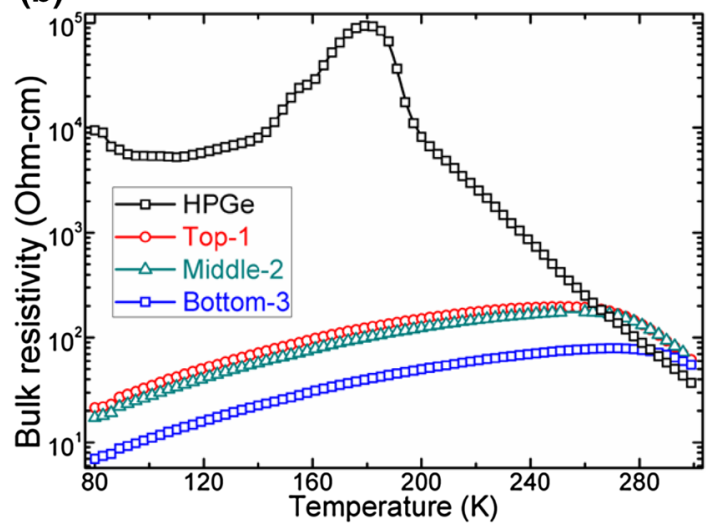

Figure 4. Temperature dependent (a) carrier mobility and (b) resistivity of HPGe and different parts of the $7 \mathrm{~N}$ pure germanium crystal.

at $77 \mathrm{~K}$. The middle and bottom parts of the crystals show an even higher carrier concentration (up to $2.2 \times 10^{13} \mathrm{~cm}^{-3}$ ) as depicted in figure $3 \mathrm{a}$. All crystals show p-type conductivity at low temperature signifying that dopants are acceptor in nature (figure $3 \mathrm{c}$ ). The carrier concentration increases with the rise in temperature due to the creation of thermally generated charge carriers. For the HPGe crystal, the thermally generated electron starts dominating around $190 \mathrm{~K}$ and the crystal shows n-type conductivity (figure 3d). Thus, extrinsic to intrinsic transition at $190 \mathrm{~K}$ is associated with the change in type of conductivity of the crystal. Pure crystals of $7 \mathrm{~N}$ with a higher acceptor concentration exhibit this extrinsic to intrinsic transition at higher temperatures (after $280 \mathrm{~K}$ ). The top and middle parts of the crystal undergo $\mathrm{p}$ - to n-type transition at 283 and $290 \mathrm{~K}$, respectively. The bottom part with the maximum acceptor concentration does not show this transition within $300 \mathrm{~K}$. It is evident that the crystal with an acceptor type impurity range of $10^{10}-10^{13} \mathrm{~cm}^{-3}$ shows p-type to n-type transition in conductivity when thermally generated intrinsic electrons dominate the acceptor dopants [24]. If the acceptor dopant concentration is higher, then the transition occurs at higher temperatures. Due to the interplay between impurity and intrinsic carriers, the resultant mobility shows a steep dip at the point when the transition in type of conductivity occurs 
(figure 4a). Bulk resistivity also shows a maximum at this transition point due to the loss in carrier mobility (figure 4b). For all the samples, carrier mobility increases at lower temperatures along with the decrease in bulk resistivity that shows maximum near intrinsic to extrinsic transition region. The ntype crystal or crystal with higher impurity concentration may not show such transition in type of conductivity [25], because the drift mobility of such a Ge crystal does not show a dip in the temperature dependent profile [26].

\section{Hall measurement of boron ion-implanted samples}

Similar phenomena have been observed in an intentionally doped HPGe crystal with varying impurity concentrations. Intentional doping is performed by boron ion implantation using the Danfysik (1080-30) ion implantation system. The ion source essentially consists of the discharge chamber and a directly heated oven which is charged with solid substances containing the material to be ionized $\left(\mathrm{BN}+\mathrm{B}_{2} \mathrm{O}_{3}\right.$ in the present case). A concentrically wound tungsten filament forms the cathode for electrode emission inside the discharge chamber. One positively charged anode ring of tungsten with a central bore is used as an inlet aperture for the source material evaporated in the oven. In the discharge space between the cathode and anode, ions are formed when electrons are emitted from the cathode, i.e., the tungsten filament. The discharge chamber on the cathode side is linked to a tungsten plate having a narrow central bore of about $0.5 \mathrm{~mm}$ diameter. Through this bore, the ions are extracted from the ion plasma by means of a highly negatively charged electrode, connected to a potential of at least $10,000 \mathrm{~V}$, and are subsequently fed to the acceleration path and the analysing magnet, respectively. Once extracted, the ions travel through a mass separator where the defined $m / q$ is selected by a sectorial magnetic field. An electrostatic scanning system is used to deflect the ion trajectories. This allows uniform irradiation of the sample (better than $3 \%$ ) with low current density (of the order of $1 \mu \mathrm{A} \mathrm{cm}^{-2}$ ). The implantation was carried out along the $\langle 100\rangle$ direction. The dose is measured by the current integrator, which integrates the current obtained from four faraday cups, using the following formula:

$$
\begin{aligned}
& \text { Dose }(Q) \\
& =\frac{[(\text { ion beam current in amps }) / q] \times[\text { implant time }]}{[\text { implant area }]} .
\end{aligned}
$$

(a)

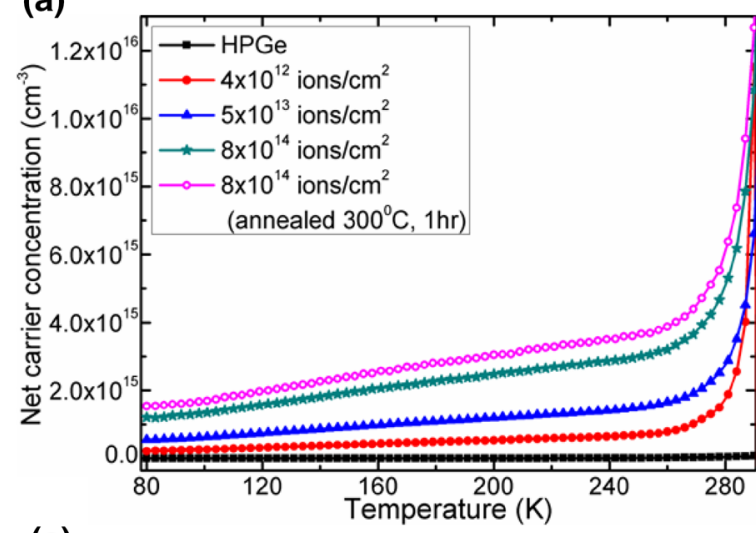

(c)

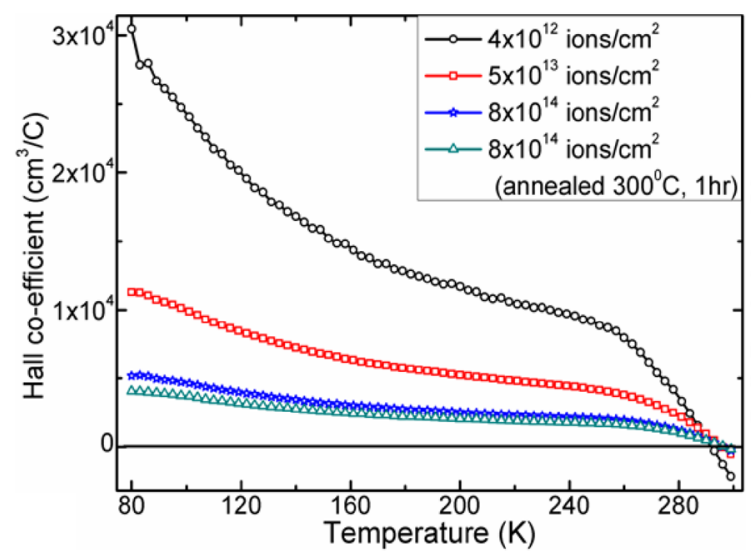

(b)

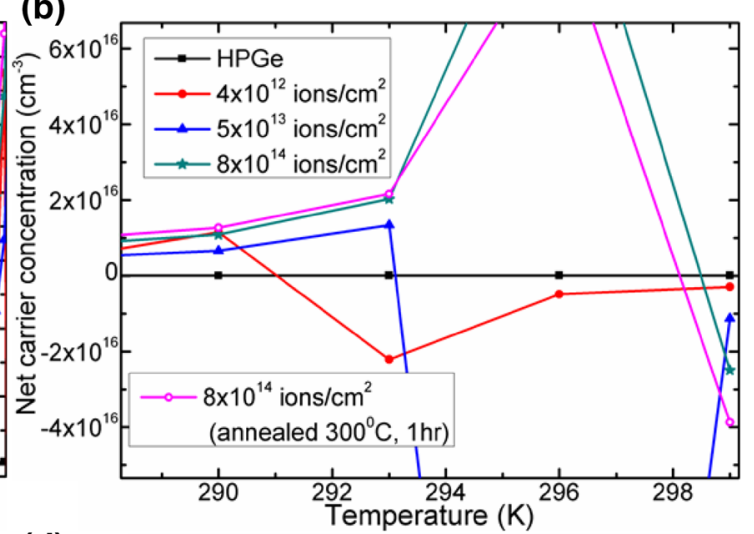

(d)

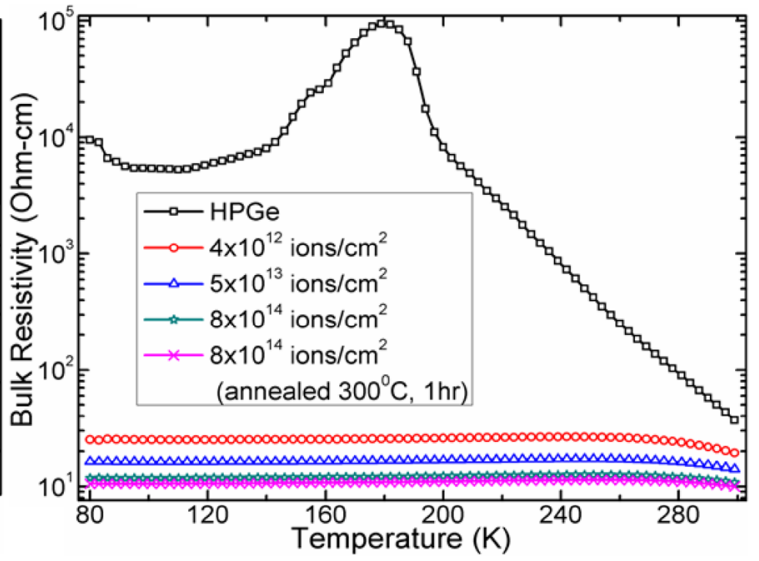

Figure 5. (a) Temperature-dependent net carrier concentration, (b) expanded view of the same near room temperature, (c) Hall coefficient and (d) bulk resistivity of the boron-implanted HPGe crystal with different doses. 


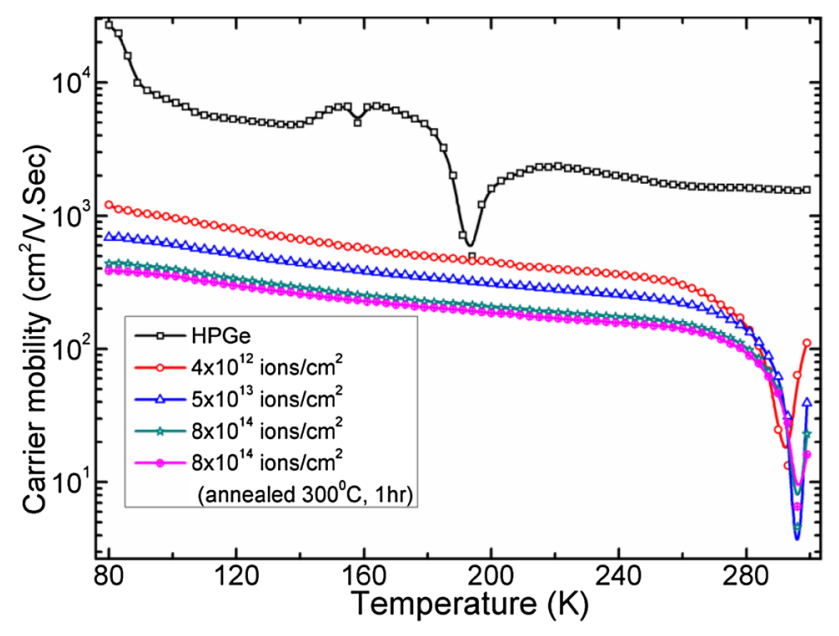

Figure 6. Carrier mobility of the boron-implanted HPGe crystal with different doses.

Temperature dependent (300-80 K) carrier concentration, hall coefficient and resistivity were obtained on ${ }^{11} \mathrm{~B}$-implanted germanium using Hall measurements in the dose range of $10^{12}-10^{14} \mathrm{~cm}^{-2}$ as shown in figure 5. The results for unimplanted HPGe are also included for comparison purposes. We also measured the influence of annealing on the electrical activity of the implanted boron ions. The net carrier concentration of the HPGe crystal increases throughout the temperature with increasing doses of implantation (figure 5a). As a result, bulk resistivity also reduces for higher boron doses (figure 5d). The carrier concentration is the highest $\left(\sim 10^{17} \mathrm{~cm}^{-3}\right)$ near room temperature due to the supply of additional carriers through thermal generation. At lower temperatures, boron-doped HPGe shows p-type conductivity. At a certain higher temperature, the thermally generated electron overpopulates the acceptor type boron impurity and the crystal exhibits n-type conductivity (figure $5 \mathrm{c}$ ). This transition occurs at higher temperatures when the boron impurity concentration increases, as seen in the case of the $7 \mathrm{~N}$ pure crystal having different impurity concentrations. The HPGe crystal with doses nearly $10^{12}, 10^{13}$ and $10^{14}$ ions per $\mathrm{cm}^{3}$ shows transition from p-type to n-type conductivity at 290, 294 and $298 \mathrm{~K}$, respectively (figure 5b and c). After implantation, annealing at $300^{\circ} \mathrm{C}$ is performed to increase the activation percentage of dopants [27]. Minor changes in electrical conduction are observed after annealing at $300^{\circ} \mathrm{C}$. Thus, room temperature electrical activation of boron in germanium is possible at the chosen implantation energy and the annealing step may be avoided to greatly reduce the risk of unwanted diffusion of electrically active metallic impurities. The depth of the boron-implanted layer in Ge is found to be $\approx 0.1 \mu \mathrm{m}$. As expected, bulk resistivity drastically reduces after boron implantation. As the boron doses increase, the resistivity gradually decreases, showing a value around $10 \Omega$ at $77 \mathrm{~K}$ for doses $8 \times 10^{14}$ ions per $\mathrm{cm}^{2}$ (figure $5 \mathrm{~d}$ ). The carrier mobility of the boron-implanted HPGe crystal with different doses also shows similar traits as seen for the $7 \mathrm{~N}$ pure Ge crystal (figure 6). Due to interplay between impurity and thermally generated carriers, the drift mobility shows a dip near the extrinsic to intrinsic transition region.

\section{Conclusions}

A large sized Ge single crystal of $7 \mathrm{~N}$ purity has been grown. The physical properties of three different parts of the crystal along the axis with varying impurity concentrations have been investigated and compared with the HPGe crystal. The top part of the grown crystal exhibits a net carrier concentration $\approx 10^{12} \mathrm{~cm}^{-3}$ that gradually descends to $\approx 10^{13} \mathrm{~cm}^{-3}$ at the bottom at $80 \mathrm{~K}$. It is found that p-type crystals having a carrier concentration below $10^{13} \mathrm{~cm}^{-3}$ exhibit extrinsic to intrinsic transition near room temperature. This transition is associated with a change in type of the crystal from p-type to n-type conductivity. The temperature, at which this conductivity 'type' transition occurs, increases with the net carrier concentration that can be considered as a mark of impurity index of the crystal. Similar phenomena have been observed in the boronimplanted HPGe crystal (carrier conc. $\sim 10^{10} \mathrm{~cm}^{-3}$ ) that further validates the trend observed in $7 \mathrm{~N}$ pure Ge crystals.

\section{Acknowledgements}

The authors are thankful to all members of the Crystal Technology Section for their unconditional help and support.

\section{References}

[1] Dalven R 1966 Infrared Phys. 6129

[2] Debye P P and Conwell E M 1953 Phys. Rev. 93693

[3] Wada K and Kimerling L C (eds) 2015 Photonics and electronics with germanium (New York: Wiley) ISBN: 978-352732821-5

[4] Hansen W L and Haller E E 1981 IEEE Trans. Nucl. Sci. NS-28 541

[5] Haller E E, Pearl P L, Hubbard G S and Hansen W L 1979 IEEE Trans. Nucl. Sci. NS-26 265

[6] Haller E E, Hansen W L and Goulding F S 1972 Nuclear Science Symposium; Miami Beach, Florida, USA, CONF721202-12 481

[7] Eberth J and Simpson J 2008 Prog. Part. Nucl. Phys. 60283

[8] Yang G, Wang G, Xiang W, Guan Y, Sun Y, Mei D et al 2012 J. Cryst. Growth 35243

[9] Haller E E, Hansen W L and Goulding F S 1981 Adv. Phys. 30 93

[10] Hubbard G S, Haller E E and Hansen W L 1978 IEEE Trans. Nucl. Sci. NS-25 362

[11] Zhang X, Friedrich S and Friedrich B 2018 J. Cryst. Process Technol. 833

[12] Yang G, Govani J, Mei H, Guan Y, Wang G, Huang M et al 2014 Cryst. Res. Technol. 49269 
[13] Robertson R and Kennett T J 1972 Nucl. Instrum. Methods 98 599

[14] Chroneos A and Bracht H 2014 Appl. Phys. Rev. 1011301

[15] Katz M J 1965 Phys. Rev. A 1401323

[16] Fritzsche H 1955 Phys. Rev. 99406

[17] Hung C S and Gliessman J R 1954 Phys. Rev. 961226

[18] Boldrini V, Maggioni G, Carturan S, Raniero W, Sgarbossa F, Milazzo R et al 2019 J. Phys. D: Appl. Phys. 52035104

[19] Spitzer W G, Firle T E, Cutler M, Shulman R G and Becker M 1955 J. Appl. Phys. 26414

[20] Nishina Y and Danielson G C 1957 Ames Laboratory Technical Reports-170, IOWA State College, ISC-926

[21] Yang G, Kooi K, Wang G, Mei H, Li Y and Mei D 2018 Appl. Phys. A 124381
[22] Yang G, Mei D, Govani J, Wang G and Khizar M 2013 Appl. Phys. A 113207

[23] Nuclear Instruments and Detectors Committee of the IEEE Nuclear and Plasma Sciences Society 1993 IEEE Std. 1160-1993, INSPEC Accession Number: 4471071

[24] Sapoval B and Hermann C 1995 Physics of semiconductors (Springer-Verlag) ISBN 978-0-387-94024-3

[25] Hung C S 1950 Phys. Rev. 79727

[26] Paige E G S 1960 J. Phys. Chem. Solids 16207

[27] Jasper C, Rubin L, Lindfors C, Jones K S and Oh J 2002 Proceedings of the International Conference on Ion Implantation Technology vol 22-27 (IEEE Inc.: USA) p 548 